Li, L., Aro, E-M., \& Millar, A. H. (2018). Mechanisms of Photodamage and Protein Turnover in Photoinhibition. Trends in Plant Science, 23(8), 667-676

\title{
Mechanisms of photodamage and protein turnover in photoinhibition
}

\section{Lei Li ${ }^{1}$, Eva-Mari Aro ${ }^{2}$, A. Harvey Millar ${ }^{1}$}

${ }^{1}$ ARC Centre of Excellence in Plant Energy Biology, School of Molecular Sciences, The University of Western Australia, 35 Stirling Hwy, 6009, Perth, Western Australia. ${ }^{2}$ Department of Biochemistry, Molecular Plant Biology, University of Turku, FI-20014, Turku, Finland.

\section{Key words}

Photodamage, protein turnover, cyclic electron flow, phosphorylation, photoprotection 


\begin{abstract}
$\underline{\text { Abstract }}$
Rapid protein degradation and replacement is an important response to photodamage and a means of photoprotection by recovering proteostasis. Protein turnover and translation efficiency studies have discovered fast turnover subunits in cytochrome $b_{6} f$ and NDH complex; in addition to PSII subunit D1. Mutations of these complexes have been linked to enhanced photodamage at least partially via cyclic electron flow. Photodamage and photoprotection involving cytochrome $b_{6} f$, NDH complex, cyclic electron flow, PSI and non-photochemical quenching proteins have been reported. We propose the rapid turnover of specific proteins in cytochrome $b_{6} f$ and the NDH complex need to be characterised and compared to inhibition of PSII by excess excitation energy and PSI by excess electron flux to expand our understanding of photoinhibition mechanisms.
\end{abstract}

\title{
$\underline{\text { Introduction }}$
}

Photosynthesis converts light energy into chemical energy in photosynthetic plants, algae and cyanobacteria. A key abiotic factor that can reduce photosynthetic capacity is 'light' itself; thus light is a substrate and an inhibitor of photosynthesis. Photoinhibition was historically described as an imbalance between PSII photodamage and its subsequent repair [1, 2]. More recently, the wider regulation of thylakoid energy transduction in different photosynthetic protein complexes has been proposed to be important for the flexibility of photosynthetic light reactions [3]. The importance of cytochrome $b_{6} f, \mathrm{NAD}(\mathrm{P}) \mathrm{H}$ dehydrogenase $(\mathrm{NDH})$ complex and cyclic electron flow around PSI in photo-acclimation have been observed in mutants with less tolerance to fluctuating light conditions [4, 5]. In addition, PSI has been reported to be inhibited by excess light via damage to its iron-sulphur clusters $\left(F_{X}\right)[6]$. However, it has been unclear whether these protein complexes are specifically targeted during photodamage or are involved in some manner during photoprotection.

In a recent protein turnover study using the model plant Arabidopsis thaliana, PetD (a subunit of the cytochrome $b_{f} f$ complex) and PIFI (an auxiliary subunit associated with the NDH complex) were shown to have comparable degradation rates to the D1 subunit of PSII. Several other plastid proteins were also found to have unexpectedly high turnover rates compared to the majority of the photosynthetic apparatus (Figure 1A, [7]). Interestingly, photosynthetic proteins including D1 in PSII, PetD in cytochrome $b_{6} f$ and NDHK in the NDH complex were also recently reported to have much faster translation rates than other comparable subunits in the same protein complexes; based on ribosome footprint profiling strategy in maize [8]. Taken together, these independent lines of evidence for proteins in photosynthetic complexes with rapid degradation and protein synthesis, raise the possibility of the involvement of previously unrecognised proteins in photodamage and repair of the plastid electron transport chain (Figure 1A). This opinion focuses on considering the evidence for the role of cytochrome $b_{6} f$ and NDH complex in photodamage and photoinhbition, while the related but more 
complex evidence for the role of cyclic electron flow around PSI in photoinhibition and PSI inhibition by excess light are outlined in Box 1 and Box 2, respectively.

\section{PSII photodamage is clearly linked to D1 degradation and repair}

The study of PSII susceptibility to photodamage was initiated by the discovery and identification of the rapid turnover of the PSII D1 subunit using radioactive labelling of proteins in isolated chloroplasts in the 1980s $[9,10]$. This early literature has been reviewed some time ago in a historical perspective on the mechanism of photoinhibition [1] and it stands as an exemplar of how a photoinhibitory mechanism can operate. The importance of D1 turnover in PSII and photosynthesis has also been highlighted by the discovery of a point mutation in Chlamydomonas D1 that resulted in even less stable PSII than wildtype and an inability of the mutant to grow photosynthetically [11]. PSII activity inhibition had been modelled as insufficient de novo synthesis of D1 to repair PSII under environmental stresses such as cold treatment [2]. Damage to PSII has been proposed to involve the disintegration of the Mn centre in PSII that then leads to an energy imbalance and a so far ill-defined oxidative damage of D1 protein residues that inactivates PSII and leads to cleavage of the D1 subunit by proteases in a two-step model [12]. An alternate proposed PSII photodamage mechanism involves elevation of the electric field component of the thylakoid proton motive force that increases PSII charge recombination rates, producing more detrimental singlet oxygen to promote PSII damage [13, 14]. Fast D1 turnover is a common feature to both these views and is central to understanding PSII photodamage and photoinhibition. Using recently developed tools for de novo discovery of high turnover rate proteins, D1 was again 'rediscovered' as a very rapidly turning over protein in both Arabidopsis and barley (Figure 1A, [7, 15]). The D1 protein has also been reported to have a much faster translation rate than other subunits in PSII in data extracted from ribosome footprint profiling in maize [8].

\section{The putative role of cytochrome $b_{6} f$ turnover in photoprotection}

Cytochrome $b_{6} f$ and PSII both contribute to the proton gradient formation between the plastidial lumen and stroma. Cytochrome $b_{6} f$ accepts electron from both LEF and CEF to control the proton gradient across the thylakoid membrane (Figure 1-2). In Chlamydomonas, which completely lacks the NDH-1 complex, the cytochrome $b_{6} f$ complex can form a supercomplex with PSI, LHCI, LHCII and ferredoxin (Fd)-NADPH oxidoreductase and control the energy balance and the mode of photosynthetic electron flow between PSII and PSI [16]. As a response to light intensity changes, cytochrome $b_{6} f$ plays a role in state transitions (processes in which LHCII balances the transfer of excitation energy to PSII and PSI) by modulating the phosphorylation states of LHCII through the antagonistic kinase and phosphatase pair of STN7 and PPH1 $[17,18]$. In higher plants, the regulation of cytochrome $b_{6} f$ through $\Delta \mathrm{pH}$ is essential for protection of PSI from light stress, which is more vulnerable to photodamage than PSII in 
the absence of cytochrome $b_{f} f[19,20]$. Because cytochrome $b_{f} f$ is needed for both LEF and CEF, it plays a key role in controlling photosynthesis and photoprotection pathways.

The stoichiometry of photosynthetic complexes need to be strictly controlled to satisfy the changing ATP and NADPH requirements of plant cells during development and environment adaptation. Protein degradation plays an important role in controlling cytochrome $b_{6} f$ amount during such conditions [21]. While its degradation has been reported to be relatively slow in higher plants in response to stress, fast degradation of cytochrome $b_{6} f$ has been observed in microalgae. In Chlamydomonas, mixotrophic growth conditions coupled with low actinic light and nitrogen starvation induced active degradation of cytochrome $b_{6} f$ and its biogenesis factors in a manner associated with CLP and FtsH family proteases [22]. Cytochrome $b_{6} f$ is the smallest photosynthetic complex with seven essential (Pet-A, B, C, D, G M, $\mathrm{N})$ and one non-essential (PetL) subunit [21]. In Arabidopsis grown under long day conditions, cytochrome $b_{6} f$ subunits Pet A-C have turnover rates similar to many other proteins in plastids, while PetD is listed amongst the fastest turning over proteins in plant leaves (Figure 1A). PetD does not stably bind any cofactors but is proposed to have a scaffold role in cytochrome $b_{\sigma} f$ and to form part of the PQ binding site together with PetB [23]. Both PetB and PetD loss-of-function mutants in tobacco are nonphotosynthetic which demonstrates their non-redundancy in cytochrome $b_{6} f$ function and photosynthesis [24]. The apparently similar roles of D1 and PetD in the PQ cycle and the similar responses of their deficiency in photoinhibition phenotypes has been recently highlighted [7]. Moreover, both PSII and cytochrome $b_{6} f$ form dimers for their functions in the PQ cycle and proton translocation. D1 and PetD are both located in the centre of their respective complexes (Figure 1A), implying that a similar photodamage and repair mechanism might be required for both proteins. The much faster turnover rate of PetD than other subunits in cytochrome $b_{6} f$ raises the question of whether it has a specific role in electron transfer and photoprotection.

\section{The putative role of NDH complex turnover in photoprotection}

The chloroplast NDH complex is a ferredoxin-dependent PQ reductase with some similarity in structure and role to the mitochondrial ETC complex I (Figure 1-2). The NDH complex functions in reduction of the PQ pool that transfers electrons to cytochrome $b_{6} f$ in CEF or to the plastid terminal oxidase (PTOX) in chlororespiration. Investigating the NDH complex in higher plants has been challenging due to its partially unresolved structure, its low abundance and its fragile properties following isolation [25]. NDH mutants typically behave almost like wild type plants but they do have a very specific postillumination chlorophyll fluorescence phenotype. Because of this, the contribution of NDH to photosynthesis was thought to be very limited [26]. However recently, the findings of selective activation of $\mathrm{NDH}$ dependent $\mathrm{CEF}$ in vivo by $\mathrm{H}_{2} \mathrm{O}_{2}[27,28]$ in addition to its physiological role 
maintaining photosynthesis under fluctuating light stress [4] support earlier proposals that NDH is involved in photoprotection $[29,30]$.

In Arabidopsis, there are 44 proteins proposed to be subunits of the NDH complex or ancillary proteins essential for NDH complex function, and some ancillary subunits have been later defined as structural subunits $[26,31]$. NDH subunits can be grouped into seven subcomplexes (A, B, M-membrane, N, EDB-electron donor binding, L-lumen and linkers) based on their functionality, position and the assembly pathway of the NDH complex $[25,26,32]$. Besides a generally similar ' $\mathrm{L}$ ' shape structure to mitochondrial complex I, subcomplex A of the NDH complex corresponds to the Complex I Q module. NDH subunits in subcomplex A (NDHK, NDHI, NDHM, NDHH), subcomplex EDB (NDHV and NDHS) and subcomplex L (PnsL5) showed relatively fast turnover rates compared to other subunits. Among these faster turning over proteins, NDHV was independently shown to stabilise the NDH complex under high light stress [33]. Subunits NDHH and NDHK, that are proposed to be the PQ binding sites on NDH complexes [34, 35], show faster turnover rates than other NDH subunits ([7];

Figure 1A). Similarly, $Q$ module proteins of mitochondria Complex I which form a matrix arm also showed significantly faster turnover rates than the proteins of the membrane arm [36, 37]. The PIFI subunit, which was found to be essential for NDH complex integrity [38], stands out as having the highest turnover rate in the NDH complex. This raises the question of its specialized role in NDH complex function. PIFI is an NDH complex ancillary protein which is conserved in higher plants but is absent in cyanobacteria, and its physical position in a NDH module has not been defined. However, it has been found to be essential for the NDH-dependent non-photochemical reduction of PQ pool, its loss-of-function leads to a change in post-illumination fluorescence and the mutant is sensitive to PSII photoinhibition [38].

\section{Experiments required to confirm or refute these new photoinhibitory mechanisms}

The systematic analysis of the turnover rate of photosynthetic proteins (Figure 1A), coupled to complex new claims about the role of CEF and PSI in photoinhibition (Box 1 and 2) has reopened old questions about the multiple ways that photoinhibition might occur. In the case of cytochrome $b_{6} f$ and NDH complexes, the new data correlate with biochemical and genetic evidence implicating them in photoinhibition. A hypothetical model can be proposed here to combine our current understanding of the effects of photodamage and photoprotection processes on photosynthetic proteins during high light stress (Figure 2). However, a systematic analysis of protein stability, abundance and functional changes under different light and photosynthetic rate conditions, post-translation protein modifications, and degradation and resynthesis of proteins coupled with measurement of LEF and CEF intensity will be necessary to establish or refute the relative importance of protein turnover rate and reversible protein complex modifications in photoinhibition (Figure 1B, Figure 2). 


\section{Investigation of multiple protein targets under photoinhibitory conditions is needed}

The most recent PSII photodamage model proposes that electrical potential plays a dominant role in modulation of PSII charge recombination rates and the subsequent production of singlet oxygen causing damage [13]. This proposal supports the importance of the ATP synthase, ion channels and transporters that can regulate thylakoid proton motive force partitioning into electric field and lumen acidification processes. It could be targeted through bioengineering in the future to enhance photoprotection $[14,39]$. This represents a new opportunity to define the mechanism of PSII specific photoinhibition and/or D1 targeted photodamage.

Unlike the D1 subunit, other rapidly degrading proteins including PetD and PIFI (Figure 1A), have not been shown to have varied stabilities when light conditions are altered or photoinhibition-sensitive mutant lines are examined. Radiolabelling was originally used to discover D1 in isolated plastids and re-examining the radiolabelled gels from those original studies indicate that D1 was the major but not the only protein band observed to be rapidly synthesised and degraded [40, 41]. Radiolabelling did not easily allow a coupling of labelling and identification for these other proteins, but today other options have become available. Firstly, ribosome footprint profiling has been used to evaluate translation events and can also be used to estimate protein synthesis rates [8,42]. Secondly, advances in analysis of metabolic labelling with stable isotopes coupled with mass spectrometry (Figure 1B) provide a robust way to explore turnover rate changes in different proteins and changes in rates for the same proteins under different treatments [43]. These two strategies used together can help to revisit and potentially discover new photodamage photosynthetic protein targets and related photodamage and photoprotection mechanisms under normal and light stress conditions.

\section{The relationship between protein phosphorylation and protein degradation should be proven}

While the role of phosphorylation in regulation of PSII mobility and repair [44, 45] as well as in regulation of LHCII-mediated interactions of the pigment protein complexes [46] have been extensively studied, we do not know if similar post-translational modifications regulate other photosynthetic complexes (Figure 1B, Figure 2). Different kinases and phosphatases contribute to the phosphorylation states of PSII and light harvesting complex II (LHCII), respectively. The damagerepair cycle of PSII is regulated through phosphorylation by STN8 kinases (Figure 2). Phosphorylation states of the D1 protein are associated with efficient D1 subunit turnover during the damagedegradation-replacement cycle. Also, another fast turnover protein, PGRL1A in CEF, has been found to be specifically phosphorylated by STN8 [47]. It will be interesting to investigate whether its phosphorylation states can affect its stability and whether its phosphorylation follow a similar pattern to D1. Changes in the plastoquinone (PQ) pool redox state can also induce state transitions $[17,18]$. STN7 kinase and PPH1 phosphatase, acting as an antagonistic pair, can play a substantial role in state 
transitions by altering LHCII phosphorylation state. STN7 is known to be active in low light but inactive in high light (Figure 2). Inactive STN7 can dock with cytochrome $b_{6} f$ through dynamic interactions with PetC in Arabidopsis [48]. This supports the early observation that the presence of cytochrome $b_{6} f$ is essential for LHCII phosphorylation [10]. Interestingly, PetC and PetD interact with each other in cytochrome $b_{\sigma} f$ complexes. In Chlamydomonas, PetD can interact with and enhance STT7 kinase autophosphorylation and a PetD phosphorylation site has been identified [49, 50]. Together these data raise the question whether PetD could be modified by phosphorylation through STN7 kinase in higher plants and whether its fast turnover rate is related to state transition stages. Just like PSII core proteins, ZEP and VDE, PIFI and PnsL5 in the NDH complex, and PetA in cytochrome $b_{6} f$ have also been found to be phosphorylated [51,52]. It will be important to define whether or not phosphorylation states affect the mobility and repair of these photosynthetic complexes, and the degradation of unstable protein targets.

\section{The role of plastid proteases in the degradation of short-lived proteins needs to be confirmed or} refuted

The contribution of plastidial proteases to efficient degradation of rapidly degrading proteins other than D1 needs to be studied (Figure 1B, Figure 2). Most proteins in the plastid are relatively stable compared to those in other compartments in a plant cell [7], but there are a range of possible mechanisms for the plastid protein degradation rates that have been observed. Chloroplasts can be damaged by UV exposure or over-accumulation of oxygen radicals and can be degraded in whole by globular vacuoles or by central vacuoles via selective autophagy transportation $[53,54]$. Specific protein degradation by selective autophagy has also been reported, but mainly for plastid stromal proteins such as RUBISCO [55]. The median degradation rates of such stromal proteins suggest that a basal degradation rate is associated with autophagy and vacuole-dependent degradation activities in the cell [7]. In contrast, degradation rates of D1, PetD and PIFI photosynthetic complexes proteins in thylakoid membrane are over five to ten times this base level. This means that degradation pathways inside plastids, rather than autophagy and the vacuole, are likely to be responsible for their selective degradation. A plastid proteolysis network, consisting of multiple classes of proteases working together, break down specific damaged proteins [56]. These proteases undertake general household proteolysis functions to maintain thylakoid protein homeostasis. CtpA and CtpA1 peptidase, CLP, DEG and FTSH family proteases have been found or proposed, to play specialised roles in efficient D1 maturation, processing and cleavage [44, 56] (Figure 2). It is expected that these proteases may also act on other fast degrading photosynthetic complex proteins such as PetD and PIFI (Figure 1-2). A parallel analysis and comparison of the stability and abundance changes of D1, PetD and PIFI in plastid proteases mutants will be an essential step to identify the proteases involved. 


\section{Resynthesis of fast degrading proteins to ensure protein homeostasis needs to be proven}

Replacement of photodamaged proteins is needed to ensure protein homeostasis and it is recognised as an essential process for plant acclimation to photoinhibitory conditions. Damaged D1 proteins in PSII are continuously replaced with newly synthesised proteins [57] (Figure 2). In plants, the D1 protein is encoded in the plastid genome by a single copy $p s b A$ gene that is highly transcribed; providing an abundant transcript pool inside plastids. The high level of $p s b A$ transcript is relatively steady over diurnal cycles. However, the transcripts are only translated to proteins in response to light and the translation is strictly regulated at the elongation step [58]. Light-stimulated translation of D1 leads to fast resynthesis that matches the fast degradation under high light (Figure 2). Like D1, PetD is encoded by the plastid genome. Ribosome footprinting in the maize plastid showed that PetD stood out amongst other cytochrome $b_{6} f$ subunits for its rapid translational rate, especially in mature green leaf segments [8]. Comparisons of transcript abundance and translation rate determined by ribosome footprinting, coupled with transcript analysis under optimal and photoinhibitory conditions are now required to settle the question of the role of PetD's translation rate (Figure 1B). In contrast, PIFI and ZEP are encoded by the nuclear genome. Protein precursors need to be first translated in the cytosol and then imported into plastids through the envelope import apparatus and sorted to the stroma and thylakoid membrane. Anterograde and retrograde signalling needs to be studied to understand the interplay between nucleus and chloroplast to keep the homeostasis of these proteins with their counterparts in protein complexes [59]. Besides the transcription and translation comparisons, changes in the rate of import for nuclear encoded products also need to be considered under different light conditions. 


\section{$\underline{\text { References }}$}

1. Adir, N. et al. (2003) Photoinhibition - a historical perspective. Photosynth Res 76, 343-70.

2. Allahverdiyeva, Y. and Aro, E.M. (2012) Photosynthetic Responses of Plants to Excess Light: Mechanisms and Conditions for Photoinhibition, Excess Energy Dissipation and Repair.

Photosynthesis: Plastid Biology, Energy Conversion and Carbon Assimilation 34, 275-297.

3. Tikkanen, M. and Aro, E.M. (2014) Integrative regulatory network of plant thylakoid energy transduction. Trends Plant Sci 19, 10-7.

4. Yamori, W. et al. (2016) A physiological role of cyclic electron transport around photosystem I in sustaining photosynthesis under fluctuating light in rice. Sci Rep 6, 20147.

5. Walker, B.J. et al. (2014) The response of cyclic electron flow around photosystem I to changes in photorespiration and nitrate assimilation. Plant Physiol 165, 453-62.

6. Tiwari, A. et al. (2016) Photodamage of iron-sulphur clusters in photosystem I induces nonphotochemical energy dissipation. Nat Plants 2, 16035.

7. Li, L. et al. (2017) Protein Degradation Rate in Arabidopsis thaliana Leaf Growth and Development. Plant Cell 29, 207-228.

8. Chotewutmontri, P. and Barkan, A. (2016) Dynamics of Chloroplast Translation during Chloroplast Differentiation in Maize. PLoS Genet 12, e1006106.

9. Metz, J.G. et al. (1983) Characterization of Nuclear Mutants of Maize Which Lack the Cytochrome-F/B-563 Complex. Plant Physiol 73, 452-459.

10. Bennett, J. et al. (1988) Cytochrome-B6f Complex Is Required for Phosphorylation of LightHarvesting Chlorophyll a/B Complex-Ii in Chloroplast Photosynthetic Membranes. Eur J Biochem $171,95-100$.

11. Hutchison, R.S. et al. (1996) Construction and characterization of a photosystem II D1 mutant (arginine-269-glycine) of Chlamydomonas reinhardtii. Biochim Biophys Acta 1277, 83-92.

12. Kato, Y. et al. (2015) D1 fragmentation in photosystem II repair caused by photo-damage of a two-step model. Photosynth Res 126, 409-16.

13. Davis, G.A. et al. (2016) Limitations to photosynthesis by proton motive force-induced photosystem II photodamage. Elife 5, doi: 10.7554/eLife.16921.

14. Davis, G.A. et al. (2017) Hacking the thylakoid proton motive force for improved photosynthesis: modulating ion flux rates that control proton motive force partitioning into Deltapsi and DeltapH. Philos Trans R Soc Lond B Biol Sci 372, doi: 10.1098/rstb.2016.0381.

15. Nelson, C.J. et al. (2014) Proteins with high turnover rate in barley leaves estimated by proteome analysis combined with in planta isotope labeling. Plant Physiol 166, 91-108.

16. Iwai, M. et al. (2010) Isolation of the elusive supercomplex that drives cyclic electron flow in photosynthesis. Nature 464, 1210-U134.

17. Minagawa, J. (2011) State transitions--the molecular remodeling of photosynthetic supercomplexes that controls energy flow in the chloroplast. Biochim Biophys Acta 1807, 897-905. 
18. Ebenhoh, O. et al. (2014) Short-term acclimation of the photosynthetic electron transfer chain to changing light: a mathematical model. Philos Trans R Soc Lond B Biol Sci 369, doi:

10.1098/rstb.2013.0223.

19. Joliot, P. and Johnson, G.N. (2011) Regulation of cyclic and linear electron flow in higher plants. Proc Natl Acad Sci U S A 108, 13317-22.

20. Colombo, M. et al. (2016) Photosynthesis Control: An underrated short-term regulatory mechanism essential for plant viability. Plant Signal Behav 11, doi: 10.1080/15592324.2016.1165382.

21. Schottler, M.A. et al. (2015) Photosynthetic complex stoichiometry dynamics in higher plants: biogenesis, function, and turnover of ATP synthase and the cytochrome b6f complex. $J$ Exp Bot 66, $2373-400$.

22. Wei, L. et al. (2014) Nitric oxide-triggered remodeling of chloroplast bioenergetics and thylakoid proteins upon nitrogen starvation in Chlamydomonas reinhardtii. Plant Cell 26, 353-72.

23. Cramer, W.A. and Zhang, H. (2006) Consequences of the structure of the cytochrome b6f complex for its charge transfer pathways. Biochim Biophys Acta 1757, 339-45.

24. Monde, R.A. et al. (2000) Post-transcriptional defects in tobacco chloroplast mutants lacking the cytochrome b(6)/f complex. Plant $J$ 21, 61-72.

25. Peng, L. et al. (2012) Multistep assembly of chloroplast NADH dehydrogenase-like subcomplex A requires several nucleus-encoded proteins, including CRR41 and CRR42, in Arabidopsis. Plant Cell 24, 202-14.

26. Shikanai, T. (2016) Chloroplast NDH: A different enzyme with a structure similar to that of respiratory NADH dehydrogenase. Biochim Biophys Acta 1857, 1015-22.

27. Strand, D.D. et al. (2015) Activation of cyclic electron flow by hydrogen peroxide in vivo. Proc Natl Acad Sci U S A 112, 5539-44.

28. Strand, D.D. et al. (2016) Defects in the Expression of Chloroplast Proteins Leads to H2O2 Accumulation and Activation of Cyclic Electron Flow around Photosystem I. Front Plant Sci 7, 2073.

29. Burrows, P.A. et al. (1998) Identification of a functional respiratory complex in chloroplasts through analysis of tobacco mutants containing disrupted plastid ndh genes. EMBOJ 17, 868-76.

30. Endo, T. et al. (1999) The role of chloroplastic NAD(P)H dehydrogenase in photoprotection. FEBS Lett 457, 5-8.

31. Suorsa, M. et al. (2009) Towards characterization of the chloroplast NAD(P)H dehydrogenase complex. Mol Plant 2, 1127-40.

32. Peng, L. et al. (2009) Efficient operation of NAD(P)H dehydrogenase requires supercomplex formation with photosystem I via minor LHCI in Arabidopsis. Plant Cell 21, 3623-40.

33. Fan, X. et al. (2015) The NdhV subunit is required to stabilize the chloroplast NADH dehydrogenase-like complex in Arabidopsis. Plant J 82, 221-31.

34. Efremov, R.G. and Sazanov, L.A. (2011) Structure of the membrane domain of respiratory complex I. Nature 476, 414-20. 
35. Battchikova, N. et al. (2011) Structure and Physiological Function of NDH-1 Complexes in Cyanobacteria. Bioenergetic Processes of Cyanobacteria: From Evolutionary Singularity to Ecological Diversity 445-467.

36. Li, L. et al. (2013) Subcomplexes of ancestral respiratory complex I subunits rapidly turn over in vivo as productive assembly intermediates in Arabidopsis. $J$ Biol Chem 288, 5707-17.

37. Li, L. et al. (2017) Changes in specific protein degradation rates in Arabidopsis thaliana reveal multiple roles of Lon1 in mitochondrial protein homeostasis. Plant J 89, 458-471.

38. Wang, D. and Portis, A.R., Jr. (2007) A novel nucleus-encoded chloroplast protein, PIFI, is involved in $\mathrm{NAD}(\mathrm{P}) \mathrm{H}$ dehydrogenase complex-mediated chlororespiratory electron transport in Arabidopsis. Plant Physiol 144, 1742-52.

39. Spetea, C. et al. (2017) An update on the regulation of photosynthesis by thylakoid ion channels and transporters in Arabidopsis. Physiol Plant 161, 16-27.

40. Ohad, I. et al. (1984) Membrane protein damage and repair: removal and replacement of inactivated 32-kilodalton polypeptides in chloroplast membranes. J Cell Biol 99, 481-5.

41. Ohad, I. et al. (1985) Light-dependent degradation of the Q(B)-protein in isolated pea thylakoids. EMBO J 4, 1655-9.

42. Juntawong, P. et al. (2014) Translational dynamics revealed by genome-wide profiling of ribosome footprints in Arabidopsis. Proc Natl Acad Sci U S A 111, E203-12.

43. Nelson, C.J. and Millar, A.H. (2015) Protein turnover in plant biology. Nat Plants 1, 15017.

44. Gururani, M.A. et al. (2015) Regulation of Photosynthesis during Abiotic Stress-Induced Photoinhibition. Mol Plant 8, 1304-20.

45. Jarvi, S. et al. (2015) Photosystem II repair in plant chloroplasts--Regulation, assisting proteins and shared components with photosystem II biogenesis. Biochim Biophys Acta 1847, 900-9.

46. Rantala, M. et al. (2017) Proteomic characterization of hierarchical megacomplex formation in Arabidopsis thylakoid membrane. Plant J 92, 951-962.

47. Reiland, S. et al. (2011) Comparative phosphoproteome profiling reveals a function of the STN8 kinase in fine-tuning of cyclic electron flow (CEF). Proc Natl Acad Sci U S A 108, 12955-60.

48. Shapiguzov, A. et al. (2016) Activation of the Stt7/STN7 Kinase through Dynamic Interactions with the Cytochrome b6f Complex. Plant Physiol 171, 82-92.

49. Grieco, M. et al. (2016) An evolutionary view on thylakoid protein phosphorylation uncovers novel phosphorylation hotspots with potential functional implications. $J$ Exp Bot 67, 3883-96.

50. Dumas, L. et al. (2017) A stromal region of cytochrome b6f subunit IV is involved in the activation of the Stt7 kinase in Chlamydomonas. Proc Natl Acad Sci U S A 114, 12063-12068.

51. Wang, P. et al. (2013) Quantitative phosphoproteomics identifies SnRK2 protein kinase substrates and reveals the effectors of abscisic acid action. Proc Natl Acad Sci U S A 110, 11205-10.

52. Zulawski, M. et al. (2013) PhosPhAt goes kinases-searchable protein kinase target information in the plant phosphorylation site database PhosPhAt. Nuc Acids Research 41, D1176-D1184. 
53. Izumi, M. et al. (2017) Entire Photodamaged Chloroplasts Are Transported to the Central Vacuole by Autophagy. Plant Cell 29, 377-394.

54. Woodson, J.D. et al. (2015) Ubiquitin facilitates a quality-control pathway that removes damaged chloroplasts. Science 350, 450-4.

55. Michaeli, S. et al. (2016) Autophagy in Plants--What's New on the Menu? Trends Plant Sci 21, 134-144.

56. van Wijk, K.J. (2015) Protein maturation and proteolysis in plant plastids, mitochondria, and peroxisomes. Annu Rev Plant Biol 66, 75-111.

57. Takahashi, S. and Badger, M.R. (2011) Photoprotection in plants: a new light on photosystem II damage. Trends Plant Sci 16, 53-60.

58. Mulo, P. et al. (2012) Strategies for psbA gene expression in cyanobacteria, green algae and higher plants: from transcription to PSII repair. Biochim Biophys Acta 1817, 247-57.

59. Dietz, K.J. et al. (2018) Toward an Integrated Understanding of Retrograde Control of Photosynthesis. Antioxid Redox Signal doi: 10.1089/ars.2018.7519.

60. Ilik, P. et al. (2017) Alternative electron transport mediated by flavodiiron proteins is operational in organisms from cyanobacteria up to gymnosperms. New Phytologist 214, 967-972.

61. Shikanai, T. and Yamamoto, H. (2017) Contribution of Cyclic and Pseudo-cyclic Electron Transport to the Formation of Proton Motive Force in Chloroplasts. Mol Plant 10, 20-29.

62. Suorsa, M. et al. (2012) PROTON GRADIENT REGULATION5 is essential for proper acclimation of Arabidopsis photosystem I to naturally and artificially fluctuating light conditions. Plant Cell 24, 2934-48.

63. Takagi, D. and Miyake, C. (2018) Proton gradient regulation 5 supports linear electron flow to oxidize photosystem I. Physiol Plant doi: 10.1111/ppl.12723.

64. Strand, D.D. et al. (2017) The higher plant plastid NAD(P)H dehydrogenase-like complex (NDH) is a high efficiency proton pump that increases ATP production by cyclic electron flow. $J$ Biol Chem $292,11850-11860$.

65. Tikkanen, M. and Grebe, S. (2018) Switching off photoprotection of photosystem I - a novel tool for gradual PSI photoinhibition. Physiol Plant 162, 156-161.

66. Mazor, Y. et al. (2017) Structure of the plant photosystem I supercomplex at 2.6 A resolution. Nat Plants 3, 17014.

67. Grieco, M. et al. (2012) Steady-state phosphorylation of light-harvesting complex II proteins preserves photosystem I under fluctuating white light. Plant Physiol 160, 1896-910.

68. Lu, Y. (2016) Identification and Roles of Photosystem II Assembly, Stability, and Repair Factors in Arabidopsis. Front Plant Sci 7, 168.

69. Kato, Y. et al. (2018) NDH-PSI Supercomplex Assembly Precedes Full Assembly of the NDH Complex in Chloroplast. Plant Physiol 176, 1728-1738.

70. Peltier, G. et al. (2016) NDH-1 and NDH-2 Plastoquinone Reductases in Oxygenic Photosynthesis. Annu Rev Plant Biol 67, 55-80. 
71. Correa-Galvis, V. et al. (2016) PsbS interactions involved in the activation of energy dissipation in Arabidopsis. Nat Plants 2, 15225.

72. Kromdijk, J. et al. (2016) Improving photosynthesis and crop productivity by accelerating recovery from photoprotection. Science $354,857-861$.

73. DalCorso, G. et al. (2008) A complex containing PGRL1 and PGR5 is involved in the switch between linear and cyclic electron flow in Arabidopsis. Cell 132, 273-285. 


\section{BOX1 Putative links between cyclic electron flow and photoprotection}

Cyclic electron flow (CEF) is an electron transfer pathway involving cytochrome $b_{6} f$, NDH and PSI (Figure 1A). But does its action lead to photoprotection? It has been proposed that CEF is required to balance the need for ATP and NADPH that are normally used at a stoichiometry of 1.5 for $\mathrm{CO}_{2}$ fixation in the Calvin-Benson cycle [60]. Cytochrome $b_{6} f$, PGR5/PGRL1, NDH and PSI can interact and form separate CEF supercomplexes that switch the mode of photosynthesis from linear electron flow (LEF) to CEF $[16,26]$. In angiosperms, it has been proposed that PGR5/PGRL1 and the NDH complex are both ferredoxin-dependent and can accept electrons from PSI and transfer them to cytochrome $b_{6} f$ through plastoquinone (PQ). The cytochrome $b_{6} f$ complex translocates protons from stroma to lumen coupled with oxidation and reduction of PQ. In cyanobacteria, algae, mosses and gymnosperms, a conserved flavodiiron protein family can transfer electrons from PSI to reduce $\mathrm{O}_{2}$ to water and this process is called the Mehler-like reaction or pseudo-CEF, and it plays an essential role in photoprotection in these species specifically [61]. In higher plants such as Arabidopsis, CEF around PSI can increase the proton motive force amplitude through the actions of cytochrome $b_{f} f$ without NADPH production. CEF can also increase in response to energy demand such as under high light and induced photorespiratory conditions of high $\mathrm{O}_{2}$ and low $\mathrm{CO}_{2}$ [5]. The potential importance of PGR5 in photoprotection has been illustrated through observations of reduction in biomass accumulation in pgr 5 mutants both under high and fluctuating light conditions [4, 62]. In addition to its role in CEF, a recent study also suggested that PGR5/PGRL1 are involved in modulation of LEF around PSI during steady state photosynthesis [63]. NDH-dependent $\mathrm{CEF}$ can be activated in vivo by $\mathrm{H}_{2} \mathrm{O}_{2}$ [27]. Plants produce excess $\mathrm{H}_{2} \mathrm{O}_{2}$ under light stress, and this may link the function of the NDH complex with photoprotection through CEF. The NDH complex pumps protons directly and increases the amplitude of the proton motive force leading to ATP production, while the PGR5/PGRL1 proteins in the putative PGRmediated electron transport pathway do not [64]. In terms of protein stability, PGR5 (At2g05620) has an average protein turnover rate in Arabidopsis leaves while PGRL1A (At4g22890) has a relatively fast protein turnover rate; approximately twice the average rate amongst major Arabidopsis leaf proteins ([7]; Figure 1A). 


\section{BOX2 PSI damage in fluctuating light has been linked to $F_{X}$ inhibition}

PSI is generally tolerant to high light excitation. However, electron flow through it is strictly controlled either by acceptance of electrons from PSII or release of electrons to CEF and to stromal metabolism (Figure 2). Frequent photodamage to PSII and dephosphorylation of LHCII under high light limit the electron flow into PSI [3]. It has been reported that this process may serve as a mechanism of PSI photoprotection against excess light. Photodamage of PSI can occur through excess electron flow-in from PSII or by a lack of electron flow-out e.g. loss-of-function of PGR5 under specific light stresses [6]. A method to induce PSI photodamage in wildtype Arabidopsis by application of controlled fluctuating light has been proposed recently [65] and this could be an useful way to investigate PSI damage and repair. Development of measurements of both electron flow through PSI and the oxidized states of the P700 reaction centre provide a means to evaluate PSI photodamage under different light stresses. In the pgr5 mutant under fluctuating light conditions, different levels of photodamage of the PSI $F_{X}$ are reflected in changes in its oxidation state [6]. Under normal growth conditions, almost all PSI proteins have been shown to have slow protein turnover rates, with the exception of PSAA ([7], Figure 1A). PSAA has been shown to contain two of the four cysteine residues ligating the $F_{X}$ cluster to the PSI complex while the terminal electron acceptors $F_{A}$ and $F_{B}$ are located in PSAC [66]. The relatively faster turnover rate of PSAA might demonstrate that some level of damage occurs to this $\mathrm{F}_{\mathrm{X}}$ protein ligand even under control conditions. Recently, measurement of $\mathrm{F}_{\mathrm{A}} / \mathrm{F}_{\mathrm{B}}$ EPR signals lost due to photodamage were reported to be partially reversible which suggests that $\mathrm{F}_{\mathrm{A}}$ and $\mathrm{F}_{\mathrm{B}}$ are able to recover, but the damaged $F_{X}$ could lead to transformation of a PSI molecule into a non-photochemical photoprotective energy quencher with high stability [6]. This may indicate a mechanism of photodamage that does not require replacement; which supports the proposal that photodamage to $\mathrm{F}_{\mathrm{X}}$ is irreversible but the protein is stable. Alternatively, plants may adjust the level of PSI excitation and function during light stress by LHCII phosphorylation [3, 67]. Phosphorylation sites were identified in higher plants PSI reaction centre proteins [49], but it is still unclear whether or not this modification plays a role in modulating PSI functionality and stability by means of protein turnover, $\mathrm{F}_{\mathrm{A}} / \mathrm{F}_{\mathrm{B}}$ or photodamaged $F_{X}$ induced PSI-NPQ transitions (Figure 1B). An in-depth analysis of PSI reaction centre protein turnover, post-translational modifications such as phosphorylation, and regulation of degradation and resynthesis under photoinhibitory conditions coupled with physiological investigations are needed to untangle if there is a direct link between PSI reaction centre photodamage and its functional transitions. 
Figure 1. Experiments required to determine whether or not the rate of degradation of photosynthetic proteins is linked to photodamage and/or photoprotection. (A) A model of photosynthetic complexes highlighting the protein turnover rate of their subunits. Positions of subunits in photosynthetic complexes are based on published annotations of PSII [45, 49, 68], Cytochrome $b_{6} f$ [49], PSI [49], NDH [26, 69, 70], ZEP/VDE [71, 72] and PGR5/PGRL1 [60, 63, 73]. Linear electron flow (LEF) is shown as solid lines. Pathways of cyclic electron flow (CEF) through NDH complex or PGR5/PGRL1 are shown as dotted lines. Protein subunits are coloured according to protein degradation rate [7]; a grey colour means no available information. (B) Current information on photodamage and the processes of replacement of proteins are divided into 'known' processes for D1 and 'experiments to assess new mechanisms' needed to confirm or refute the role of newly discovered unstable proteins in photodamage and replacement. Steps needed to further investigate if PSI subunits undergo photodamage, reactivation and selective replacement are also proposed.

(A)

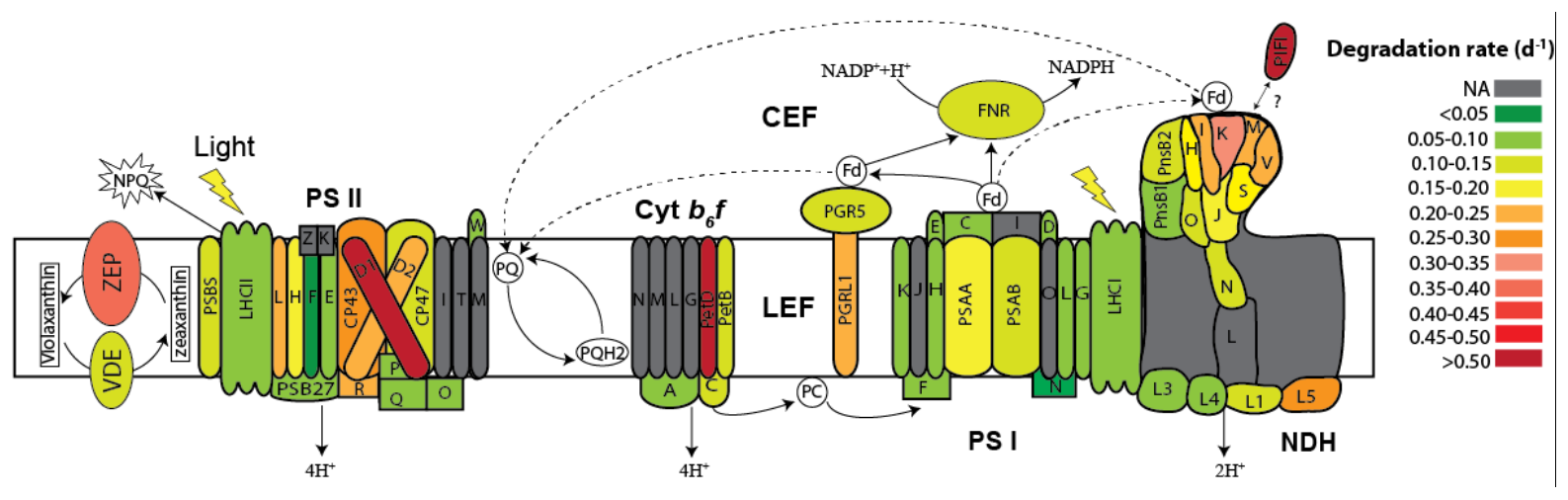

(B)

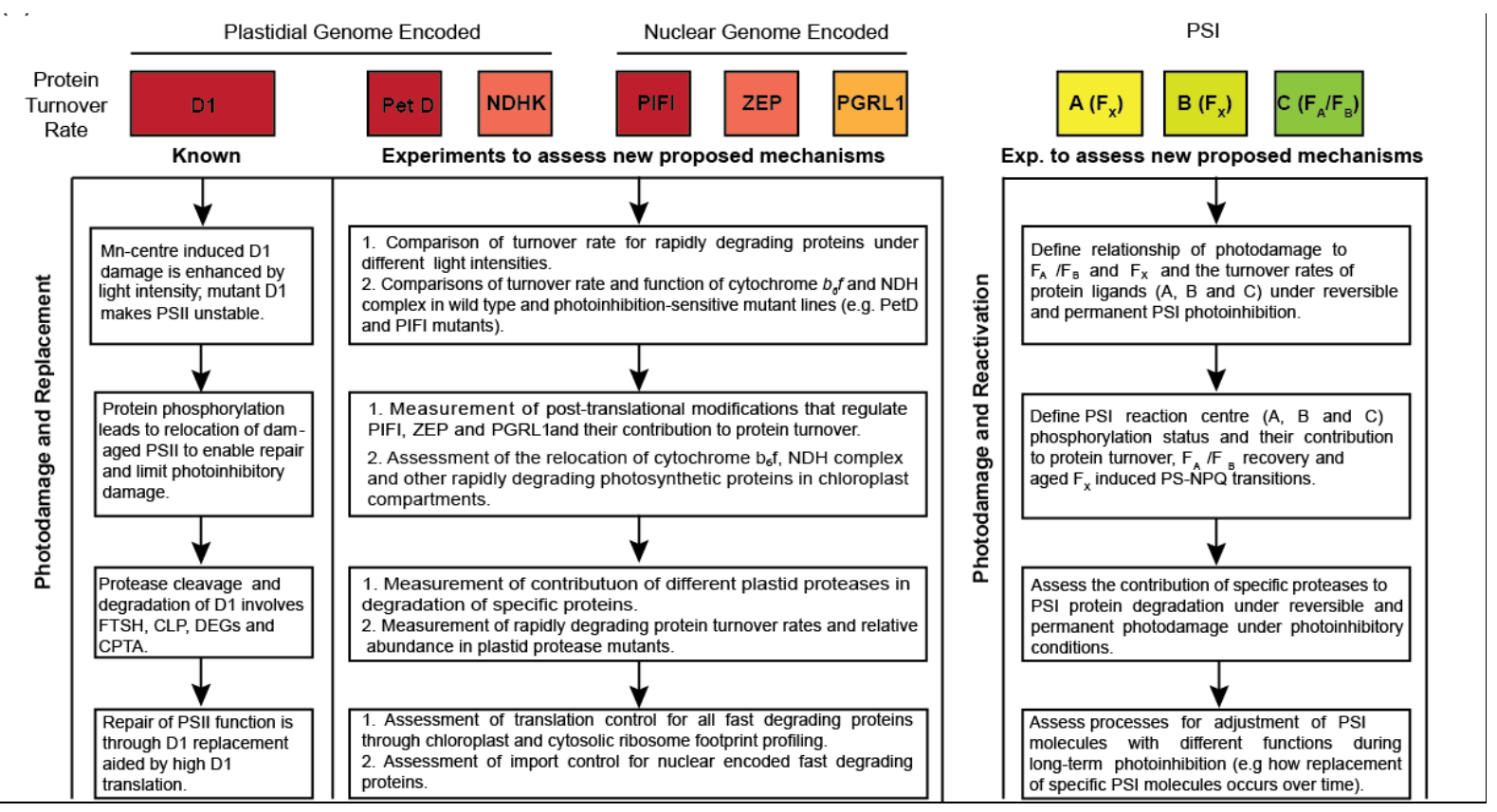


Figure 2. A hypothetical model of how photosynthetic protein turnover and changes in electron flow could combine in plant cells under different light regimes. The diagram illustrates electron flow rate, NPQ activity, and degradation and synthesis of photodamaged proteins under low and high light conditions with protein turnover rate annotated by colour (as in Figure 1A). High light stress can result in more zeaxanthin and a higher level of NPQ. The rate of both LEF and CEF would increase in high light leading to higher $\Delta \mathrm{pH}$ between thylakoid lumen and stroma. The acidification of thylakoid lumen is essential for formation of active protease complexes todegrade photodamaged D1 (e.g. DEG and FTSH complex formation) and the proton gradient also activates resynthesis of D1 through translational control at the elongation step [45]. Kinase STN8 is activated by high light and increases the phosphorylation level of PSII core proteins while high light inhibits the phosphorylation of LHCII by the STN7 kinase. The phosphorylation state of LHCII, D1 and other photosynthetic proteins modify LHCII-PSII and PSI interactions and photodamaged protein replacement. Complete dephosphorylation of LHCII under high light prevents the excess excitation energy transfer to PSI. Higher $\Delta \mathrm{pH}$ also allows more ATP production through ATP synthase for protein degradation and synthesis. Upon long term high light acclimation, plants can increase the relative abundance of PSI to PSII, restore their redox states and diminish the susceptibility to photodamage [3]. These photoprotective mechanisms would give PSI a high tolerance to high light stresses and higher overall protein stability. To maintain fast turnover protein homeostasis, rapid degradation of damaged proteins would be required via proteases in chloroplasts. Chloroplast proteases located in the stroma (CLP/DEG7), thylakoid membrane (FtsH), and lumen (DEG) are involved in degradation of D1 and may also be involved in degradation of other fast turnover photosynthetic proteins. Gene expression in the nucleus and chloroplast genomes, translational control and import control will need to be coordinated for rapid replacement of proteins induced by light stress.

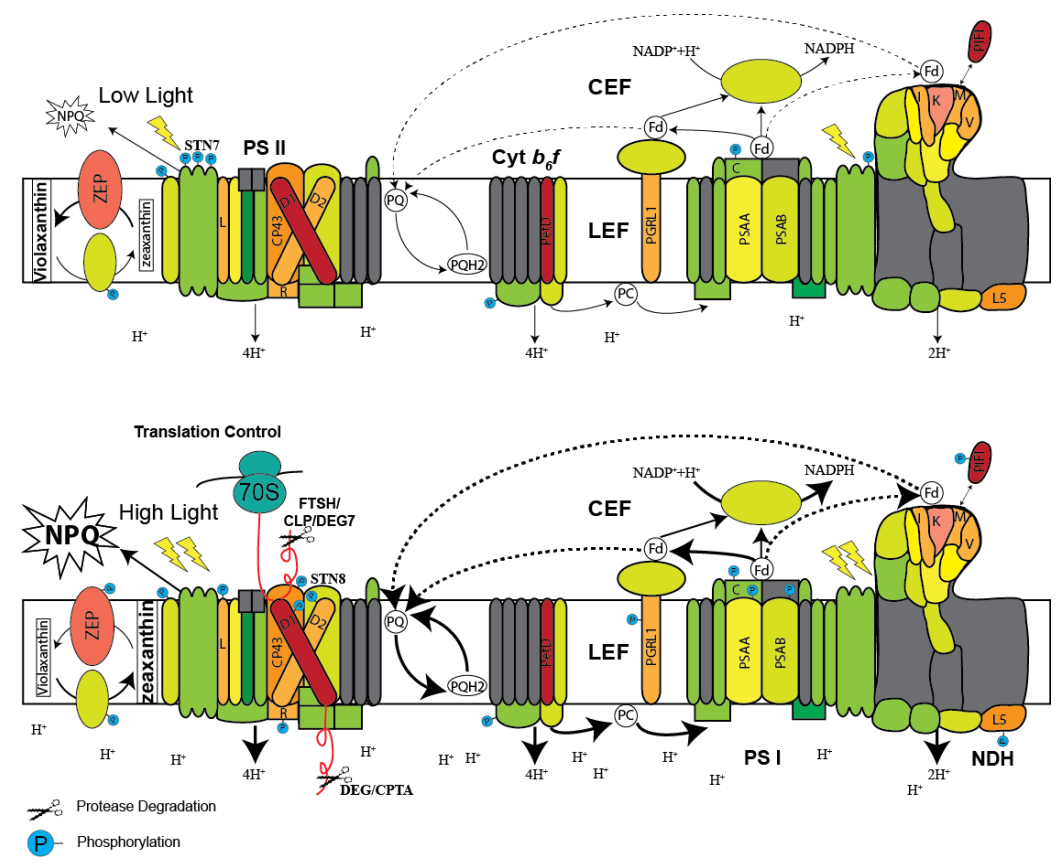




\section{Glossary}

Protein turnover

Protein turnover is the combination of degradation and synthesis of specific proteins that maintains the steady state abundance of each protein in a cell. It maintains protein quality and function throughout different stages of growth and development. It is a high energy cost to cells; representing at least $25 \%$ of all cellular ATP use.

Photodamage

Photodamage is used here to describe modifications to protein properties that contribute to the decrease in photosynthesis efficiency through the actions of light. High irradiance, fluctuating light and short wavelength light can each cause different levels or types of damage to photosynthetic proteins which lead to photosynthesis efficiency reduction. Plants are equipped with different photoprotection mechanisms to limit photodamage and overcome its effects on photosynthetic rate. 